\title{
CATETERES INTRAVENOSOS FRATURADOS: RETIRADA POR TÉCNICAS ENDOVASCULARES*
}

\author{
Gustavo Andrade ${ }^{1}$, Romero Marques ${ }^{1}$, Norma Brito ${ }^{1}$, Ângelo Bomfim ${ }^{1}$, Douglas Cavalcanti ${ }^{2}$, \\ Carlos Abath ${ }^{1}$
}

Resumo OBJETIVO: A colocação de cateter para acesso venoso central é uma prática médica cada vez mais comum, sendo a fratura e embolização de fragmentos do cateter raras, porém correspondem aos corpos estranhos intravasculares mais comuns. $O$ objetivo é demonstrar nossa experiência na retirada desses corpos estranhos intravasculares utilizando técnicas endovasculares. MATERIAIS E MÉTODOS: Análise retrospectiva dos últimos cinco anos permitiu a avaliação de dez casos consecutivos, com a idade variando entre nove meses e 67 anos. RESULTADOS: 0 procedimento foi realizado com sucesso em todos os casos, por diferentes técnicas, sem complicações. Os locais mais comuns de alojamento dos fragmentos foram átrio direito, veia cava superior e artéria pulmonar esquerda. A retirada desses corpos estranhos por técnicas endovasculares é procedimento relativamente simples quando comparado à alternativa cirúrgica, tendo sido utilizada com segurança e sucesso em inúmeros pacientes. Os dispositivos disponíveis mostraram-se bastante eficazes, sendo o laço o mais versátil. CONCLUSÃO: A alta taxa de sucesso com poucas complicações relatadas, mesmo em crianças, permite a afirmação que os corpos estranhos intravenosos devem ser extraídos por técnicas percutâneas sempre que possível. Contudo, a familiarização com as diversas técnicas é fundamental, permitindo combinações e modificações, adaptando-as à situação do caso.

Unitermos: Vias de acesso vascular; Corpos estranhos; Embolia.

Abstract Intravenous catheter fragments: endovascular retrieval.

OBJECTIVE: Central venous access is an increasingly frequent procedure and intravenous catheter fractures and fragments embolization, although being rare, correspond to the most common intravascular foreign bodies. This study purpose is to show our experience in the removal of these foreign bodies, employing endovascular techniques. MATERIALS AND METHODS: Retrospective analysis of ten consecutive cases in the last five years, including patients with ages ranging from 9 months to 67 years. RESULTS: The procedure was successfully performed in all the cases by means of different techniques and with no complication. Most common fragments lodgement sites were: right atrium, superior vena cava and left pulmonary artery. The retrieval of these foreign bodies by means of endovascular techniques is a relatively simple procedure when compared to the surgical alternative, and has been safely and successfully performed in countless patients. The available devices have proven quite effective and, among them, the loop snare is the most versatile. CONCLUSION: The high success rate with few complications reported, even in children, allows us to say that, whenever possible, percutaneous extraction of intravascular foreign objects should be performed. Notwithstanding, familiarization with the several techniques available is essential, allowing combinations and modifications according to each situation.

Keywords: Vascular access ports; Foreign bodies; Embolism.

\section{INTRODUÇÃO}

A obtenção de acesso venoso central é prática crescente na medicina, sendo rara a embolização desses cateteres ou fragmentos, o que representa apenas cerca de $1 \%$ das complicações ${ }^{(\mathbf{1}-3)}$. Apesar dessa pequena incidência, o grande número de aces-

* Trabalho realizado na Angiorad - Grupo de Radiologia Inter vencionista, Recife, PE.

1. Médicos Radiologistas Intervencionistas da Angiorad.

2. Cirurgião Vascular da Angiorad.

Endereço para correspondência: Dr. Gustavo Andrade. Angiorad. Avenida Agamenon Magalhães, 2291, sala 302. Recife, PE, 50100-010. E-mail: gustavoandrade@angiorad.com.br

Recebido para publicação em 25/5/2005. Aceito, após revisão, em 2/9/2005. sos venosos centrais torna-os responsáveis pela enorme maioria de corpos estranhos intravenosos ${ }^{(1)}$.

O objetivo deste estudo é demonstrar nossa experiência na retirada de corpos estranhos intravenosos, utilizando técnicas endovasculares, nos últimos cinco anos (dez pacientes).

\section{MATERIAIS E MÉTODOS}

Entre fevereiro de 1999 e março de 2004, um grupo de dez pacientes consecutivos é descrito, com variação na idade entre 9 meses e 67 anos (Tabela 1). Todos os procedimentos de colocação do cateter de acesso venoso central foram realizados por cirurgiões, sem orientação por imagem.

A fratura do cateter ocorre, geralmente, durante a inserção, porém em um caso ocorreu na retirada (Tabela 1 - paciente 8 ; Figura 3). Uma vez percebida a fratura e confirmada por radiografia do tórax, o paciente era encaminhado ao nosso serviço de radiologia intervencionista em caráter emergencial.

\section{RESULTADOS}

Todos os procedimentos foram realizados em sala de angiografia sob acompanhamento anestésico. Através de punção 
Tabela 1 Ordem cronológica dos dez pacientes consecutivos submetidos à retirada percutânea de fragmento fraturado de cateter venoso, incluídos neste estudo.

\begin{tabular}{|c|c|c|c|l|l|l|l|}
\hline$N^{\circ}$ & Sexo & Ano & Idade & Inserção cateter & Alojamento do corpo estranho intravenoso & Acesso/retirada & \multicolumn{1}{|c|}{ Dispositivo(s) } \\
\hline 1 & M & 1999 & 63 anos & Subclávia direita & Veia cava superior, átrio direito & Femoral direita & Basket \\
2 & M & 1999 & 67 anos & Subclávia direita & Subclávia direita, veia cava superior & Femoral direita & Basket \\
3 & F & 1999 & 51 anos & Subclávia direita & Veia cava superior, átrio direito & Femoral direita & Basket \\
4 & M & 2000 & 60 anos & Subclávia esquerda & Subclávia esquerda, veia cava superior & Femoral direita & Basket \\
5 & M & 2000 & 9 meses & Jugular direita & Veia cava superior, átrio direito & Femoral bilateral & Pig-tail e loop-snare \\
6 & F & 2001 & 49 anos & Subclávia esquerda & Subclávia esquerda, veia cava superior & Femoral direita & Loop-snare \\
7 & F & 2002 & 41 anos & Jugular direita & Veia cava superior, átrio direito & Femoral direita & Basket \\
8 & F & 2002 & 2 anos & Subclávia esquerda & Veia cava superior, átrio direito & Femoral direita & Loop-snare \\
9 & F & 2003 & 58 anos & Subclávia direita & Ventrículo direiro, pulmonar esquerda & Femoral bilateral & Pig-tail e loop-snare \\
10 & M & 2004 & 32 anos & Jugular direita & Pulmonar esquerda & Femoral direita & Loop-snare \\
\hline
\end{tabular}

$\mathrm{M}$, masculino; F, feminino.

venosa percutânea femoral (direita ou bilateral), um introdutor 8F (French) permitia o manejo dos diferentes materiais que se fizessem necessários. Um cateter prémoldado 5F e um fio-guia de troca (260 $\mathrm{cm}$ ) eram conduzidos até posicionar-se o fio-guia adjacente ao fragmento de cateter (corpo estranho). $\mathrm{O}$ cateter $5 \mathrm{~F}$ era então retirado e o dispositivo desejado - cesta (Figura 1) ou laço (Figura 3) - era introduzido sobre o fio-guia. Neste momento o corpo estranho intravenoso era capturado pelo dispositivo, sendo então tracionado e retirado.

Obtivemos sucesso técnico em todos os casos, sem complicações. Nenhum procedimento durou mais de 40 minutos, entre a punção e a retirada.

Em dois pacientes (Tabela 1 - pacientes 5 e 9; Figuras 2 e 4), pela ausência de extremidade livre do fragmento, foi necessária a mobilização inicial do fragmento com um cateter "pig-tail", trazendo o fragmento a uma posição mais favorável que permitisse a retirada com o laço (Snare Amplatz; Microvena, White Bear Lake, MN).

\section{DISCUSSÃO}

Fratura de cateteres de acesso venoso central é complicação rara, porém séria, podendo levar a tromboembolia, sepse, arritmia cardíaca, efusão pericárdica, lesão miocárdica e endocardite bacteriana. Corpos estranhos intravasculares não retirados podem ocasionar complicações sérias ou fatais em até $71 \%$ dos pacientes ${ }^{(\mathbf{1})}$, com mortalidade variando entre $24 \%$ e $60 \%^{(4)}$. Apesar de alguns pacientes permanecerem

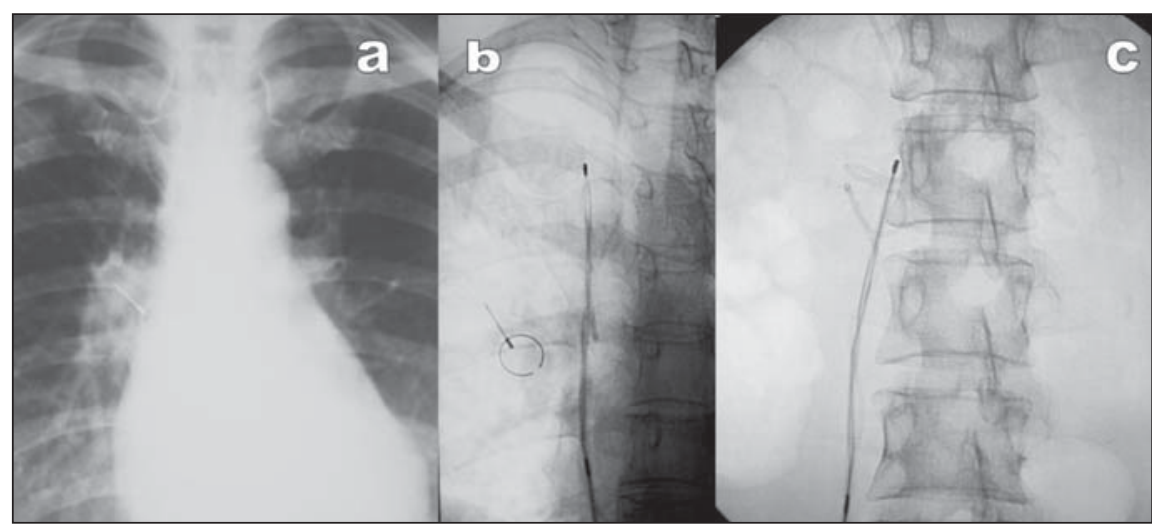

Figura 1. A radiografia de tórax mostra o "port" à direita e o cateter livre na topografia da veia subclávia direita, cava superior e átrio direito (a). Uma cesta foi utilizada para a captura do cateter no átrio direito (b), sendo o conjunto extraído (c) pela veia femoral comum direita.

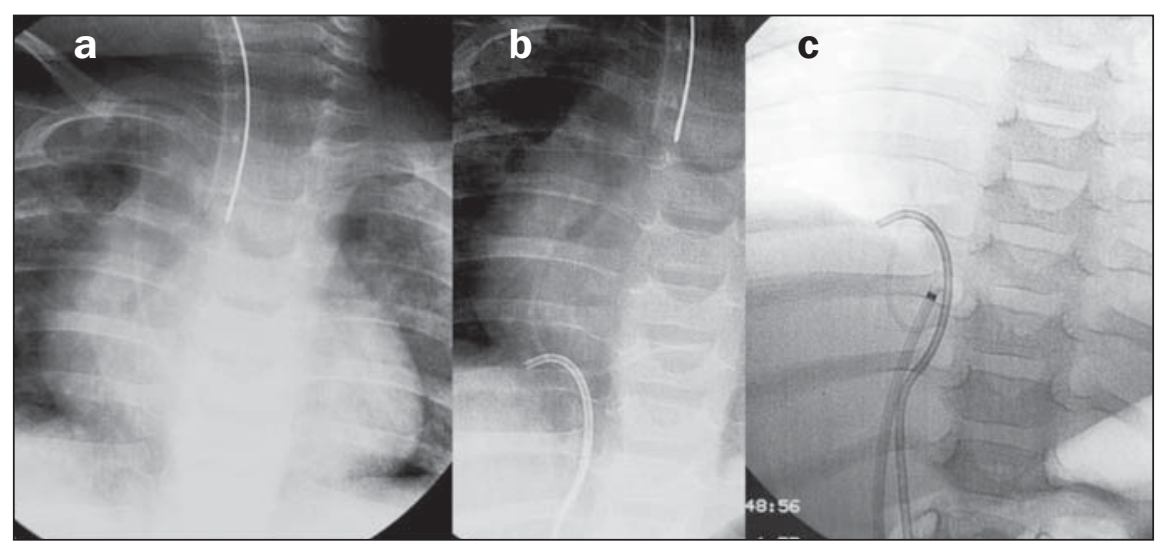

Figura 2. Um fragmento de cateter é visualizado na topografia do tronco venoso braquiocefálico direito e veia cava superior (a). Por meio de acesso pela veia femoral direita, um cateter "pig-tail" 5F foi enganchado no fragmento e tracionado, trazendo o fragmento para a veia cava inferior (b), onde uma extremidade livre foi capturada por um laço $(\mathbf{c})$.

completamente assintomáticos, o risco dessas complicações torna a remoção sempre desejada.

A retirada percutânea de corpos estranhos intravenosos é um procedimento re- lativamente simples quando comparada à opção cirúrgica, tendo sido segura e efetiva em inúmeros pacientes, mesmo em crianças e pré-termos ${ }^{(1-11)}$. O incidente transprocedimento mais comumente relatado é ar- 
ritmia cardíaca, que é sempre transitória e relacionada à manipulação intracardíaca. Esses fragmentos geralmente podem ser retirados atraumaticamente com alta taxa de sucesso, tendo o laço ("loop snare") provado ser o dispositivo mais eficaz e o preferido $^{(\mathbf{1 - 1 3})}$. Nosso grupo, inicialmente, optava pela cesta ("basket") (Figura 1), quando não dispúnhamos de laços industrialmente produzidos. Uma vez disponíveis, no ano 2000, é fácil observar a mudança no dispositivo preferido (Tabela 1), sendo o laço, atualmente, nossa primeira opção (Figura 3).

Os sítios mais comuns de alojamento dos cateteres fraturados são o átrio direito, a veia cava superior e a artéria pulmonar esquerda $^{(1-4)}$, sendo o átrio direito e a veia cava superior as localizações mais observadas nesta série (Tabela 1). Nós sempre utilizamos um cateter diagnóstico $5 \mathrm{~F}$ prémoldado e um fio-guia longo para chegarmos adjacente ao corpo estranho intravenoso, para então, utilizando o guia, colocarmos o dispositivo (laço ou cesta) na posição desejada. Uma vez capturado o fragmento, por serem flexíveis e dobráveis, podem ser retirados pela bainha introdutora 8F. Quando o fragmento encontra-se numa posição distante (Figura 4) ou com ambas as extremidades em contato com a parede do vaso (Figura 2), é muito difícil ou até impossível capturá-lo apenas com um laço. Nesta situação, um cateter "pig-tail" 5F pode ser utilizado para enroscar-se em torno do fragmento, puxando-o para uma posição melhor (Figuras 2 e 4). Com a utilização dessas abordagens, removemos com sucesso todos os fragmentos, porém, quando necessário, outras variações técnicas podem ser utilizadas.

As técnicas intervencionistas para extração de corpos estranhos intravasculares sofreram mudanças significativas com o passar dos anos. Atualmente, vários são os dispositivos disponíveis, como os laços, as cestas, os balões, o fórceps, os cateteres pré-moldados, os guias direcionáveis e os magnetos. Todos estes provaram ser bastante efetivos em determinadas situações, sendo os laços os mais versáteis ${ }^{(\mathbf{1 - 1 3})}$. A alça do laço tem o ângulo estabelecido em relação ao cabo $\left(90^{\circ}\right)$, e os laços são disponíveis numa grande variedade de tamanhos para o ajuste ideal no vaso ${ }^{(\mathbf{1 2})}$.

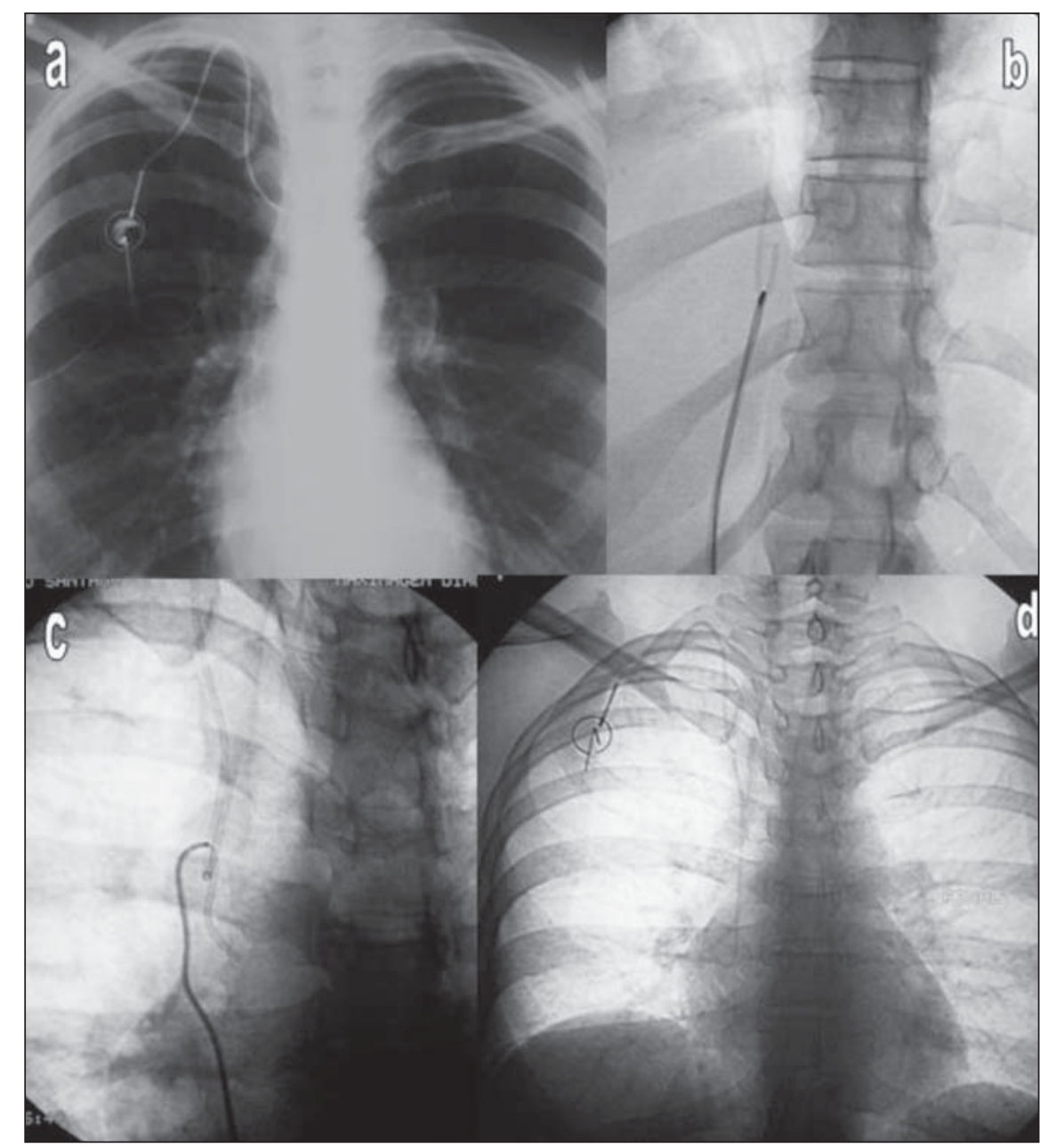

Figura 3. O paciente foi submetido a troca de cateter tipo "port" da subclávia esquerda para a jugular direita. 0 cirurgião nos encaminhou o paciente porque o cateter esquerdo não foi retirado. A radiografia do tórax com injeção de contraste no "port" direito mostra que este está no tronco venoso braquiocefálico esquerdo e que há um corpo estranho na veia cava superior (a). Um laço foi utilizado para pescar e retirar o corpo estranho (b). Com um cateter Mikaelson 5F (c), o cateter mal posicionado foi colocado na posição adequada (d).

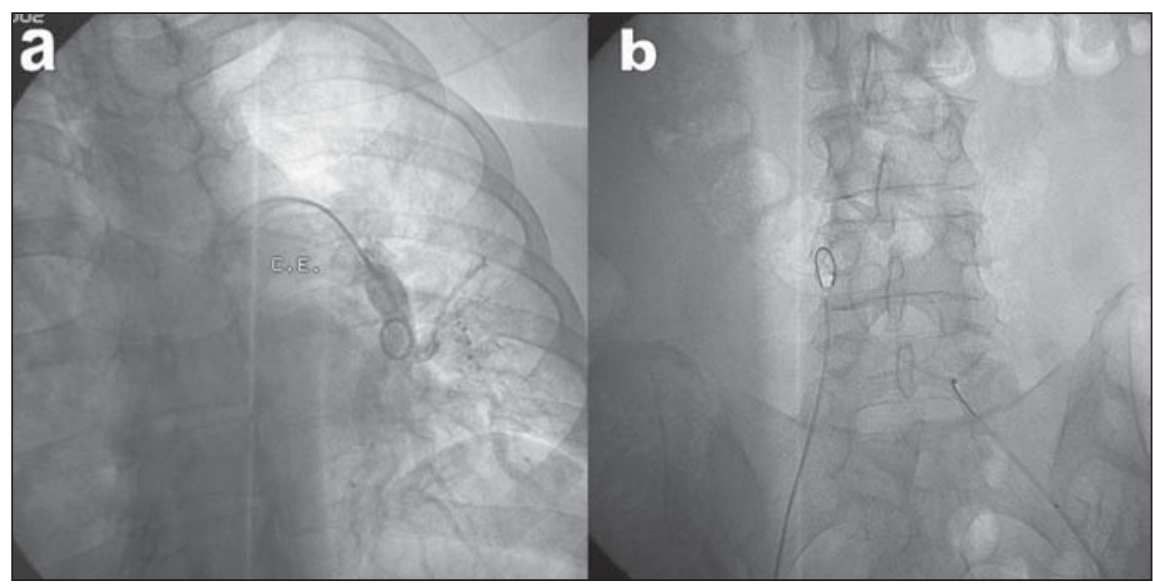

Figura 4. Há um fragmento de cateter na artéria pulmonar esquerda, onde um "pig-tail" $5 \mathrm{~F}$ está sendo usado para enroscar-se e trazer o corpo estranho para a cava inferior (a). Neste momento, um laço introduzido pela femoral contralateral é utilizado para capturá-lo e retirá-lo (b). 


\section{CONCLUSÃO}

A alta taxa de sucesso, com pouquíssimas complicações, mesmo em crianças, permite a afirmação que os corpos estranhos intravasculares devem ser extraídos, sempre que possível, por técnicas percutâneas. Para isto, é fundamental a disponibilidade dos materiais, bem como a familiarização do médico operador com as diversas técnicas e materiais, permitindo-o combiná-las e modificá-las, adaptando-as à situação. Contudo, algumas vezes, a criatividade é essencial.

\section{REFERÊNCIAS}

1. Smouse HB, Fox PF, Brady TM, Swischuk JL Castañeda F, Pham MT. Intravascular foreign body removal. Semin Intervent Radiol 2000;17 201-212.
2. Andrews RE, Tulloh RM, Rigby ML. Percutaneous retrieval of central venous catheter fragments. Arch Dis Child 2002;87:149-150.

3. Uflacker R, Lima S, Melichar AC. Intravascular foreign bodies: percutaneous retrieval. Radiology 1986;160:731-735

4. Gabelmann A, Kramer S, Gorich J. Percutaneous retrieval of lost or misplaced intravascular objects. AJR Am J Roentgenol 2001;176:1509_ 1513.

5. Hehir DJ, Cross KS, Kirkham R, Moore DJ, Shanik DG. Foreign body complications of central venous catheterisation in critically ill patients. Ir J Med Sci 1992;161:49-51.

6. Huang CH, Chen WJ, Ho YL, Wu CC, Lee YT Nonsurgical transvenous retrieval of fractured implantable central venous access device. J Formos Med Assoc 1999;98:265-270.

7. Chow LM, Friedman JN, Macarthur C, et al. Peripherally inserted central catheter (PICC) fracture and embolization in the pediatric population. J Pediatr 2003;142:141-144.

8. Liu JC, Tseng HS, Chen CY, et al. Percutaneous retrieval of intravascular foreign bodies: experi- ence with 19 cases. Kaohsiung J Med Sci 2002; 18:492-499.

9. Egglin TK, Dickey KW, Rosenblatt M, Pollak JS. Retrieval of intravascular foreign bodies: experience in 32 cases. AJR Am J Roentgenol 1995; 164:1259-1264.

10. Cekirge S, Weiss JP, Foster RG, Neiman HL, McLean GK. Percutaneous retrieval of foreign bodies: experience with the nitinol goose neck snare. J Vasc Intervent Radiol 1993;4:805-810.

11. Yang FS, Ohta I, Chiang HJ, Lin JC, Shih SL, Ma YC. Non-surgical retrieval of intravascular foreign body: experience of 12 cases. Eur J Radiol 1994;18:1-5.

12. Yedlicka JW Jr, Carlson JE, Hunter DW, Castañeda-Zuniga WR, Amplatz K. Nitinol goose neck snare for removal of foreign bodies: experimental study and clinical evaluation. Radiology 1991;178:691-693.

13. Casserly IP, Goldstein JA, Rogers JH, Lasala JM Paradoxical embolization of a fractured guidewire: successful retrieval from left atrium using a snare device. Catheter Cardiovasc Interv 2002, $57: 34-38$. 